\title{
Type Synthesis of 2T1R Decoupled Parallel Mechanisms Based on Lie Groups and Screw Theory
}

\author{
Shuwei Qu, ${ }^{1}$ Ruiqin $\mathrm{Li}^{1}{ }^{1}$ and Shaoping Bai ${ }^{1,2}$ \\ ${ }^{1}$ School of Mechanical and Power Engineering, North University of China, Taiyuan 030051, China \\ ${ }^{2}$ Department of Mechanical and Manufacturing Engineering, Aalborg University, Aalborg, Denmark \\ Correspondence should be addressed to Ruiqin Li; liruiqin@nuc.edu.cn
}

Received 2 March 2017; Revised 17 May 2017; Accepted 22 May 2017; Published 5 July 2017

Academic Editor: Yuri Vladimirovich Mikhlin

Copyright (c) 2017 Shuwei Qu et al. This is an open access article distributed under the Creative Commons Attribution License, which permits unrestricted use, distribution, and reproduction in any medium, provided the original work is properly cited.

\begin{abstract}
Decoupled parallel mechanisms (DPMs) have the characteristics of compact structure and simple control with wide applications. This paper presents a new method of type synthesis for DPMs by virtue of Lie groups and screw theory. The method consists of synthesis at limb level and configuration level. At limb level, Lie group is used to synthesize the limbs with required DOFs. At configuration level, screw theory is adopted to determine configuration with synthesized limbs that satisfy the type synthesis criteria of DPMs. The type synthesis criteria including limb decoupling and selection of the driving pairs are presented. Upon the formulation, the procedure of type synthesis of DPMs is developed. Type synthesis is conducted with the proposed method, which leads to new spatial and planar fully decoupled 2T1R mechanisms.
\end{abstract}

\section{Introduction}

Parallel mechanisms consist of two or more limbs in parallel, which bring PMs the advantage of high stiffness, high accuracy, low inertia, and high dynamic performances. On the other hand the coupling of the parallel limbs implies some problems, such as high nonlinearity relationship between input and output, which makes static and dynamic analysis and the robot control difficult [1]. Decoupled parallel mechanisms (DPMs) have one-to-one correspondence relationship between input and output variables, becoming desirable for both robot design and control.

Many techniques and approaches for DPMs type synthesis can be found in literature, for example, type synthesis of 3-DOF translational DPMs [2], T3R2 manipulators [3], and T2R1 bifurcated planar-spatial manipulators [4], in which linear transformation method was used. A 2R1T partially decoupled manipulator was proposed in [5]. Examples of synthesis with screw theories can be found in [6-12]. Moreover, 3-DOFs translational DPMs [13] and 6-DOFs decoupled manipulators [14] were synthesized with basic revolute kinematic pairs. Other examples of DPMs can be found in [15-18].
This work is interested in lower-mobility mechanisms with simple structures. In particular, 2T1R PMs generates one rotation (1R) and two translations (2T). This type of mechanisms has very wide applications in industry, such as flight motion simulation, pointing and tracking, and assembling and machining $[4,12,19,20]$, while type synthesis was generally studied for other types of parallel manipulators. Type synthesis of 2T1R DPMs was not systematically addressed.

In this work, we propose a systematic approach of $2 \mathrm{~T} 1 \mathrm{R}$ DPM synthesis. It is noted that most type synthesis was based on screw theory $[6-12,19,20]$, which does not consider the instantaneous motion characteristics. We incorporate Lie groups in synthesis, taking advantage of Lie groups in describing mathematically the precise succession of motions $[21,22]$.

The paper is organized as follows. The principle of type synthesis of topological conditions is presented in Section 2. The decoupling identification of PMs, synthesis criteria of the limb decoupling, the selection criteria of the driving pairs, and the procedure of type synthesis for DPMs are established in Section 3. The specific synthesis process and 
the configuration of 2T1R DPMs are presented in Section 4. A brief analysis of the decoupled motion is provided in Section 5. Section 6 concludes this work.

\section{Basic Principle of Type Synthesis}

Type synthesis of DPMs can be generalized into three basic problems, namely, (1) identifying topological conditions and calculating the number of structural parameters; (2) determining type synthesis method; (3) designing limbs with desired properties, assembling limbs, and obtaining mechanisms, as further explained presently.

2.1. Topological Conditions of Type Synthesis for DPMs. DPMs consist of a moving platform connected to the base by at least two limbs. The output characteristics of the moving platform are the intersection of terminal kinematic characteristics of all limbs [23-26]. That is,

$$
S_{\mathrm{DPM}}=S_{L 1} \cap S_{L 2} \cap \cdots \cap S_{L i} \cdots \cap S_{L n}
$$

where $S_{\mathrm{DPM}}$ is the output characteristics of the moving platform and $S_{L i}$ is the kinematic characteristics of $i$ th limb $(i=1,2, \ldots, n)$.

Equation (1) represents the relationship between fundamental topology elements of a mechanism. The structure parameters contain the dimension of the moving platform, the number of limbs, actuated limbs, driving pairs, redundant limbs, and so forth. Therefore, the topological structure of DPMs and the structure parameters must satisfy the following conditions $[23,27]$

$$
\begin{aligned}
F_{D}-\sum_{i=1}^{m} q_{i} & =0, \\
N & =F_{D}-\sum_{i=1}^{m}\left(q_{i}-1\right)+n=m+n, \\
m & \leq F_{D}, \\
q_{i} & \leq F_{D} \quad(i=1,2, \ldots, m),
\end{aligned}
$$

where $F_{D}$ is the dimension characteristics of the moving platform for parallel topologies, $q_{i}$ is the number of driving pairs in actuated limb $i, m$ is the number of actuated limbs, $N$ is the number of limbs, and $n$ is the number of the redundant limbs.

2.2. Type Synthesis Method Based on Screw Theory. Assume the expected number of degrees of freedom for the DPM to be $M(w, p)$, where $w$ and $p$ denote the number and the property of the degree of freedom required by the DPM, respectively. Based on screw theory [25], the screw system of the mechanism is $\left\{\oiint_{M} \mid\left(\oiint_{1}^{m}, \oiint_{2}^{m}, \ldots, \oint_{w}^{m}\right)\right\}$. This means that if $\oiint_{M}$ is known, $M(w, p)$ can be determined. By solving the reciprocal screw of $\phi_{M}$, the reciprocal screws of the screw system of the mechanism can be determined. The reciprocal screws of the screw system of the limbs $\left\{\oiint_{r}^{l j} \mid\left(\oiint_{j 1}^{l r}, \oiint_{j 2}^{l r}, \ldots, \oiint_{j i}^{d r}\right)\right\}$ (where $i \leq 6-w$ is the number of the reciprocal screws of the $j$ th limb) are a subset of those of the mechanism, which can be expressed as

$$
\left\{\oiint_{j 1}^{l r}, \oiint_{j 2}^{l r}, \ldots, \oiint_{j i}^{l r}\right\} \subseteq\left\{\oiint_{1}^{r}, \oiint_{2}^{r}, \ldots, \oiint_{6-w}^{r}\right\} .
$$

If the mechanism consists of $k$ limbs, the set composed of the reciprocal screws of the screw system of all limbs is equal to the set composed of those of the mechanism, which is

$$
\bigcup_{j=1}^{k}\left\{\phi_{j 1}^{l r}, \oint_{j 2}^{l r}, \ldots, \oint_{j i}^{l r}\right\}=\left\{\oiint_{1}^{r}, \oiint_{2}^{r}, \ldots, \oint_{6-w}^{r}\right\} .
$$

Furthermore, the reciprocal screws of the screw system and the screw systems of the limbs can be determined. The linear combination of the screw system of the limbs can constitute different types of kinematic limbs. These kinematic limbs are assembled according to certain relationships into mechanisms; if the obtained mechanism can realize continuous movement and its DOF remain invariable, then it is just the DPM which possesses the given DOF.

The entire process of the constrained screw synthesis method can be described as follows:

$$
\begin{gathered}
M(w, p) \Leftrightarrow \\
\left\{\oiint_{M} \mid\left(\oiint_{1}^{m}, \oiint_{2}^{m}, \ldots, \oiint_{w}^{m}\right)\right\} \Leftrightarrow \\
\left\{\oiint_{r} \mid\left(\oiint_{1}^{r}, \oiint_{2}^{r}, \ldots, \oiint_{6-w}^{r}\right)\right\} \Leftrightarrow \\
\left\{\oiint_{r}^{l j} \mid\left(\oiint_{j 1}^{l r}, \oiint_{j 2}^{l r}, \ldots, \oiint_{j i}^{l r}\right)\right\} \Leftrightarrow \\
\left\{\oiint_{m}^{l j} \mid\left(\oiint_{j 1}^{l m}, \oiint_{j 2}^{l m}, \ldots, \oiint_{j(6-i)}^{l m}\right)\right\} \Leftrightarrow
\end{gathered}
$$

$\{\mathrm{PMs}\}$.

Equation (5) must satisfy the following conditions:

$$
\begin{array}{r}
\left\{\oiint_{j 1}^{l r}, \oiint_{j 2}^{l r}, \ldots, \oiint_{j i}^{l r}\right\} \subseteq\left\{\oiint_{1}^{r}, \oiint_{2}^{r}, \ldots, \oiint_{6-w}^{r}\right\}, \\
\bigcup_{j=1}^{k}\left\{\oiint_{j 1}^{l r}, \oiint_{j 2}^{l r}, \ldots, \oiint_{j i}^{l r}\right\}=\left\{\oiint_{1}^{r}, \oiint_{2}^{r}, \ldots, \oiint_{6-w}^{r}\right\}, \\
\bigcap_{j=1}^{k}\left\{\oiint_{j 1}^{l m}, \oiint_{j 2}^{l m}, \ldots, \oiint_{j(6-i)}^{d m}\right\}=\left\{\oiint_{1}^{m}, \oiint_{2}^{m}, \ldots, \oiint_{w}^{m}\right\} .
\end{array}
$$

Equation (6) indicates that the reciprocal screws of the screw system of the limbs are the subset of those of the mechanism, the reciprocal screws of the screw system of the mechanism is the union of those of all limbs, and the screw system of the mechanism is the intersection of those of all limbs.

2.3. Limb Synthesis. In order to generate continuous movement, desired limbs are synthesized through Lie groups, which describe the continuous motion using precise mathematical model.

The motion characteristics fulfilling the algebra structures of Lie groups are represented by 12 kinds of displacement subgroups. Other motion characteristics not fulfilling 
the algebra structures of Lie groups are represented by displacement submanifolds. Hervé [21] enumerated all 12 kinds of displacement subgroups. The main properties are as follows: the intersection of subgroup follows the rule of intersection of sets. The intersection of two subgroups is always a subgroup. The product is also displacement subgroups, and their product can commute to each other, the product of two same displacement subgroups is always equivalent to this subgroup.

Limb synthesis is conducted through combining various kinematic pairs. The mathematical model and the topology structure parameters of limb are developed with specified motion characteristics. The kinematic pair type and quantity are identified based on the motion characteristics. The position and direction of kinematic pairs are identified according to the relationship between the joint axes.

\section{Type Synthesis Method of DPMs}

\subsection{Decoupling Identification}

3.1.1. The Decoupling Identification of Parallel Mechanism. In $\mathrm{PMs}$, the moving platform moves either in $2 \mathrm{D}$ or in $3 \mathrm{D}$ space, actuated by driving pairs. Its position and orientation are specified by a suitable set of $n_{s} \leq 6$ coordinates $S$ while the motion of the driving pairs is represented by a suitable set of $n_{q}$ coordinates $Q$. The input-output relations are represented by the forward $F(\cdot)$ and inverse $G(\cdot)$ kinematic relations

$$
\begin{aligned}
& S=F(Q), \\
& Q=G(S) .
\end{aligned}
$$

Differentiating (7) with respect to time, the velocity equation is

$$
\dot{S}=J_{a} \dot{Q}
$$

Thus,

$$
V=J_{a} \dot{Q}
$$

where $(\cdot)$ is derivative of the time, $J_{a}$ is $n \times n$ Jacobian matrix, $V$ is the linear or angular velocity of the moving platform.

According to the condition of the Jacobian matrix [3], three types of PMs are identified as follows:

(i) The PM is isotropic, if $J_{a}$ is a diagonal matrix with identical diagonal elements.

(ii) The PM is decoupled, if $J_{a}$ is a diagonal matrix with different diagonal elements or a triangular matrix.

(iii) The PM is coupled, if $J_{a}$ is neither a triangular nor a diagonal matrix.

3.1.2. Decoupling. Each output of DPMs is controlled by one or a group of inputs. Each coordinate $\left(s_{i}\right)(i=1,2, \ldots, 6)$ of the reference point of the moving platform depends on single

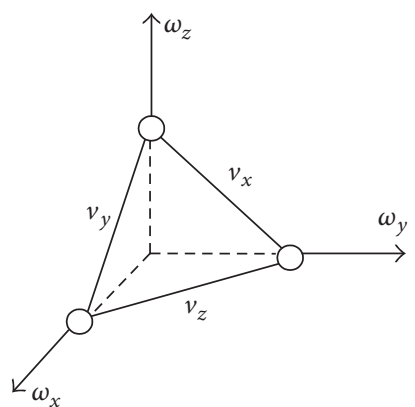

FIGURE 1: Canonical configuration of the twist.

or a group of driving pairs $\left(q_{i}\right)(i=1,2, \ldots, 6)$, the expression being given as follows:

$$
S=F(Q)=\left\{\begin{array}{l}
s_{1}=f_{1}\left(q_{1}\right) \\
s_{2}=f_{2}\left(q_{1}, q_{2}\right) \\
\vdots \\
s_{6}=f_{6}\left(q_{1}, q_{2}, q_{3}, q_{4}, q_{5}, q_{6}\right) .
\end{array}\right.
$$

3.2. Type Synthesis of DPMs. Decoupled motion of parallel mechanisms means the motion characteristics of the moving platform satisfies canonical configuration in all directions. Based on screw theory, the active twist must be linearly independent with other twists of the limb. The twist of the moving platform of parallel mechanism in general form is

$$
S=\left(\omega_{x}, \omega_{y}, \omega_{z} ; v_{x}, v_{y}, v_{z}\right)
$$

where $\omega=\left(\omega_{x}, \omega_{y}, \omega_{z}\right)$ is the angular velocity and $v=$ $\left(v_{x}, v_{y}, v_{z}\right)$ is the linear velocity.

The canonical configuration means that the axes of the revolute pairs must be orthogonal to each other and the directions of the prismatic pairs must be perpendicular to each other. The canonical configuration can be represented as infinity tetrahedron, as shown in Figure 1 [25].

The realized conditions of decoupled movement of the moving platform depend on two factors: the configuration of the limb and the selection criterion of the driving pairs.

3.2.1. Type Synthesis Criteria of the Limb Decoupling. The kinematic characteristics of the limb can be expressed by the exponential product equation. That is,

$$
g_{\mathrm{ST}}(\theta)=e^{\theta_{1} \bar{\xi}_{1}} e^{\theta_{2} \bar{\xi}_{2}} \cdots e^{\theta_{i} \bar{\xi}_{i}} \cdots e^{\theta_{n} \bar{\xi}_{n}} g_{\mathrm{ST}}(0),
$$

where $g_{\mathrm{ST}}(\theta)$ is the position of the limb with respect to $\theta$, $\xi_{i}$ is axis coordinate of identity screw of the $i$ th joint $(i=$ $1,2, \ldots, n), \theta_{i}$ is motion parameters of $i$ th joint, and $g_{\mathrm{ST}}(0)$ is initial position of the limb.

When $\xi_{i}$ is a revolute pair, $\theta_{i} \in \mathbb{S}^{1}$ is a revolute angle of $i$ th joint. When $\xi_{i}$ is a prismatic pair, $\theta_{i} \in R$ is a displacement of $i$ th joint. 
Equation (12) shows the calculation is more simple and quick if the revolute pair is located next to the moving platform. This is more useful in limb design.

The independent limb synthesis depends on the dimensional constraint of the limb [3]. In order to realize orthogonal constraints, the limbs must synthesize in orthogonal configuration. The kinematic pairs also must be synthesized in orthogonal conditions, which are as follows:

(1) The number of limbs could be determined according to topological conditions of (2).

(2) In different directions, the prismatic pairs must be perpendicular to each other.

(3) The axes of the revolute pairs must be orthogonal or parallel to each other.

(4) The axes of the revolute pairs must be perpendicular with the prismatic pairs.

(5) The prismatic pairs should be placed close to the base.

(6) The last kinematic pair in the kinematic chain of all limbs must be revolute pair if the output of the moving platform has rotation movement requirement. The axes of all revolute pairs must be parallel with direction of the rotation of the moving platform.

3.2.2. Selection Criteria of the Driving Pairs. The selection of driving pairs must follow the following criteria:

(1) The number of driving pairs should be determined according to topological conditions of (2).

(2) The DOF of the mechanism must be zero when the driving pairs are locked.

(3) The driving pairs should be distributed among all limbs as evenly as possible. It can be conveniently controlled as expressed in (10).

(4) In order to reduce the inertia of the mechanism, the driving pairs should preferably be placed on the base or close to the base.

3.3. Procedure of the Type Synthesis for DPMs. The procedure of type synthesis using Lie groups and screw theory can be summarized as follows:

(1) Identifying the topological conditions, defining the number of limbs and the structure parameters.

(2) Generating the equivalent limbs according to required DOF of DPMs by Lie groups.

(3) Distributing the corresponding constraint screw for each limb according to type synthesis method of the reciprocal screws of the screw system for DPMs.

(4) Selecting limbs from the equivalent limbs to satisfy the synthesis criteria of limb decoupling and the criteria of the driving pairs.

(5) Classifying the limbs from Step (4) according to the reciprocal screws of the screw system of Step (3). Different mobility limbs were obtained.
(6) Generating different mobility decoupled limbs, configuring DPMs.

The procedure of type synthesis for DPMs is presented in Figure 2.

\section{Type Synthesis of 2T1R DPMs}

The characteristics of the moving platform of 2T1R DPMs are two translational DOFs and one rotational DOF. The rotational axis of the revolute DOF can be parallel or perpendicular to the plane composed of the translational axes. Therefore, the type synthesis of DPMs should be discussed separately. This work focuses on the parallel situation and the result for the vertical situation will be presented directly.

4.1. Topological Condition of 2T1R DPMs. The characteristics of the moving platform of 2T1R DPMs is

$$
\left(\omega_{x}, 0,0 ; v_{x}, v_{y}, 0\right) \text {. }
$$

The intersection algorithm was employed as follows:

$$
\begin{aligned}
\left(\omega_{x}, 0,0 ; v_{x}, v_{y}, 0\right)= & \left(\omega_{x}, 0,0 ; v_{x}, v_{y}, 0\right) \\
& \cap\left(\omega_{x}, \omega_{y}, 0 ; v_{x}, v_{y}, 0\right) \\
& \cap\left(\omega_{x}, \omega_{y}, \omega_{z} ; v_{x}, v_{y}, 0\right) \\
& \cap\left(\omega_{x}, 0, \omega_{z} ; v_{x}, v_{y}, 0\right) \\
& \cap\left(\omega_{x}, \omega_{y}, 0 ; v_{x}, v_{y}, v_{z}\right) \\
& \cap\left(\omega_{x}, 0, \omega_{z} ; v_{x}, v_{y}, v_{z}\right) \\
& \cap\left(\omega_{x}, \omega_{y}, \omega_{z} ; v_{x}, v_{y}, v_{z}\right) .
\end{aligned}
$$

According to the parameters relationship of (2), the structure parameters can be expressed as follows:

$$
\begin{gathered}
F_{D}=3, \\
N=3, \\
m=3, \\
q_{i}=1, \\
n=0 .
\end{gathered}
$$

4.2. Synthesis of Equivalent Limbs of 2T1R DPMs Based on Lie Groups. The moving platform of DPMs with 2T1R movements can be expressed as semidirect product of two translations and one rotation of Lie groups [26]:

$$
\mathrm{SE}(3)=\mathrm{SO}(2) \otimes T(2) \text {. }
$$

The displacement subgroup is

$$
g(\omega)=\left\{\left(\begin{array}{cc}
e^{\theta \widehat{\omega}} & \alpha \mathbf{u}+\beta \mathbf{v} \\
0 & 1
\end{array}\right) \begin{array}{c}
\theta \in[0,2 \pi] \\
\alpha, \beta \in R
\end{array}\right\},
$$

where $\omega=R z, \mathbf{u}, \mathbf{v} \perp \omega$ or $\mathbf{u} \| \omega$ or $\mathbf{v} \| \omega$. 


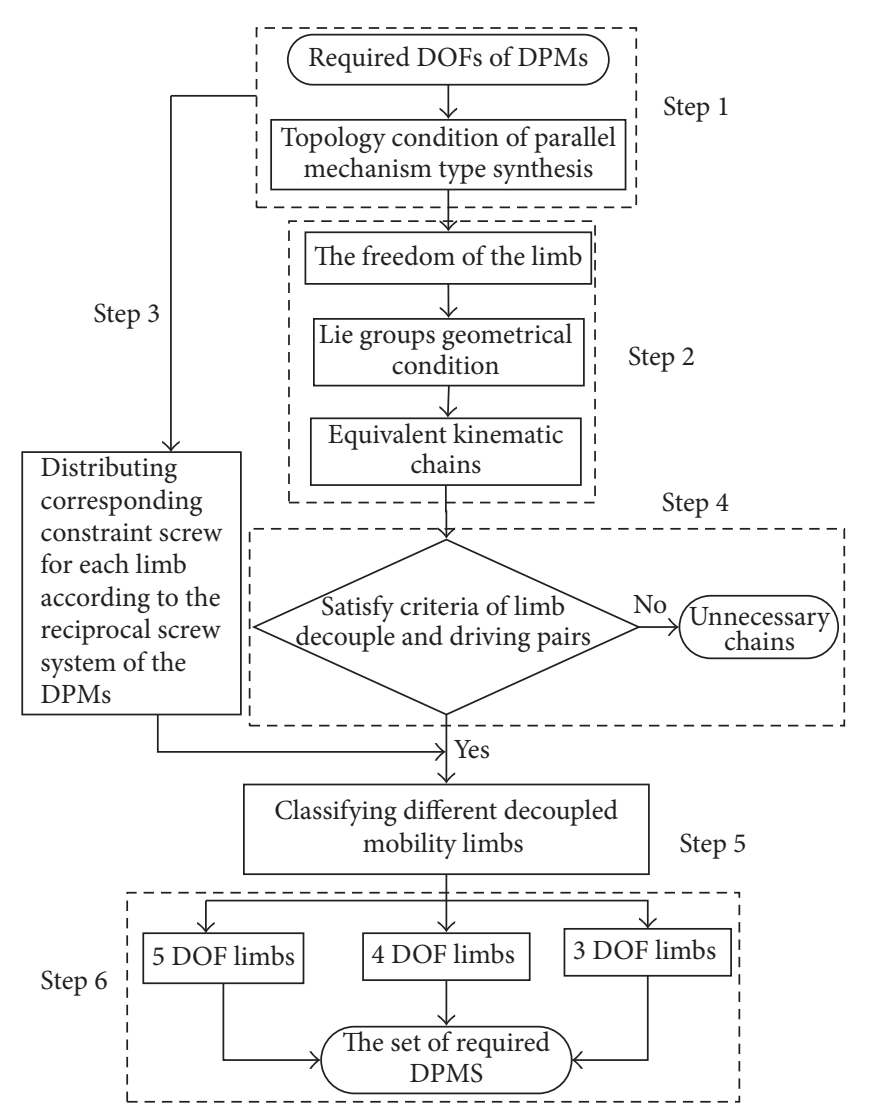

FIgure 2: Procedure of type synthesis for DPMs.

The transformed canonical configuration is as follows:

$$
g(z)=\left\{\left(\begin{array}{cc}
e^{\theta \bar{z}} & \alpha x+\beta y \\
0 & 1
\end{array}\right) \begin{array}{c}
\theta \in[0,2 \pi] \\
\alpha, \beta \in R
\end{array}\right\} .
$$

Generated canonical configuration submanifold is

$$
T_{2}\left(Z^{n}\right) \cdot R(N, x)=T_{2}\left(\omega_{1}^{n}\right) \cdot R\left(N, \omega_{2}\right) .
$$

From (16) (19), the Lie groups conditions of 2T1R DPMs are

$$
\begin{aligned}
T_{2}(x) \cdot R(N, y) & =T_{2}(x) \cdot \mu(N, x, y)=D_{2}^{3}(N, x, y) \\
& =g(x) \cdot R(N, y)=\chi(y) .
\end{aligned}
$$

Based on (20) generate the equivalent limbs, as shown in Table 1.

4.3. Distributing Constraint Screw for Different Limbs Based on Screw Theory. The screw system of 2T1R DPMs is

$$
\oiint_{M}=\left\{\begin{array}{l}
\oiint_{1}^{m}=(1,0,0 ; 0,0,0) \\
\oiint_{2}^{m}=(0,0,0 ; 1,0,0) \\
\oiint_{3}^{m}=(0,0,0 ; 0,1,0) .
\end{array}\right.
$$

The reciprocal screw system is

$$
\oiint_{r}=\left\{\begin{array}{l}
\oiint_{1}^{r}=(0,0,1 ; 0,0,0) \\
\oiint_{2}^{r}=(0,0,0 ; 0,1,0) \\
\oiint_{3}^{r}=(0,0,0 ; 0,0,1) .
\end{array}\right.
$$

According to screw theory, there are two constraint couples and one constraint force. The limbs reciprocal screw system is as follows:

(1) The DOF of limb is 5, which contains one constraint force, and the other limbs with constraint force must be parallel with the first force in space.

(2) The DOF of limb is 4, which contains one constraint couple and one constraint force. The constraint force is parallel with the constraint force of the first limb.

(3) The DOF of limb is 3, which contains two constraint couples and one constraint force. All couples of limbs must be noncoplanar in space and not parallel in space. The constraint force is parallel with the other two limbs and coplanar in space. 
TABLE 1: The equivalent limbs of 2T1R PMs.

\begin{tabular}{|c|c|}
\hline Generator & Equivalent limbs \\
\hline$T_{2}(x) \cdot R(N, y)$ & {$\left[{ }^{z} P^{y} P\right]^{y} R,{ }^{z} P^{y} C,{ }^{z} P^{y} R^{y} P,\left[{ }^{z} P^{y} P_{a}\right]^{y} R,\left[{ }^{z} P_{a}^{y} P\right]{ }^{y} R,\left[{ }^{z} P_{a}^{y} C\right],{ }^{z} P_{a}^{y} R^{y} P,\left[{ }^{z} P_{a}^{y} P_{a}\right]^{y} R$} \\
\hline $\begin{array}{l}T_{2}(x) \\
\mu(N, x, y)\end{array}$ & $\begin{array}{l}{\left[{ }^{x} P^{y} P^{z} P^{y} R^{z} R\right],\left[{ }^{x} P^{y} P^{z} P_{a}^{y} R^{z} R\right],\left[{ }^{x} P^{y} P_{a}{ }^{z} P_{a}^{y} R^{z} R\right],\left[{ }^{x} P_{a}^{y} P_{a}{ }^{z} P_{a}^{y} R^{z} R\right],\left[{ }^{x} P^{y} P^{z} P^{y z} U\right],\left[{ }^{x} P^{y} P^{z} P_{a}^{y z} U\right],\left[{ }^{x} P^{y} C^{z} C\right],} \\
{\left[{ }^{x} P^{y} P_{a}^{z} P_{a}^{y z} U\right],\left[{ }^{x} P_{a}^{y} P_{a}^{z} P_{a}^{y z} U\right],\left[{ }^{x} P_{a}^{y} C^{z} C\right]}\end{array}$ \\
\hline$D_{2}^{3}(N, x, y)$ & 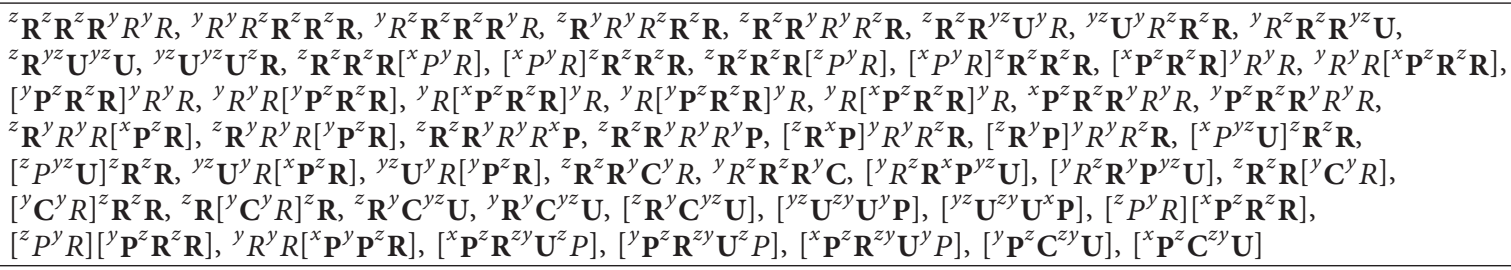 \\
\hline$g(x) \cdot R(N, y)$ & $\begin{array}{l}{ }^{x} \mathbf{R}^{x} \mathbf{R}^{x} \mathbf{R}^{y} R,{ }^{x} \mathbf{R}^{x} \mathbf{R}^{x} \mathbf{R}^{x y} \mathbf{U},\left[{ }^{x} \mathbf{R}^{z} \mathbf{P}\right]^{x y} \mathbf{U},\left[{ }^{x} \mathbf{R}^{x} \mathbf{R}^{z} \mathbf{P}\right]^{y} R,\left[{ }^{x} \mathbf{R}^{x} \mathbf{R}^{y} \mathbf{P}\right]^{y} R,{ }^{x} \mathbf{R}^{x} \mathbf{R}^{z} \mathbf{C}, \\
{ }^{x} \mathbf{R}^{x} \mathbf{R}^{y} R^{y} P,{ }^{x} \mathbf{R}^{x y} \mathbf{U}^{y} \mathbf{P},{ }^{z} \mathbf{P}^{x y} \mathbf{U}^{y} \mathbf{P},\left[{ }^{x} \mathbf{R}^{y} \mathbf{P}^{z} \mathbf{P}\right]^{y} R,\left[{ }^{x} \mathbf{R}^{z} \mathbf{P}\right]^{y} R^{y} \mathbf{P},{ }^{x} \mathbf{R}^{z} \mathbf{P}^{y} \mathbf{C},\left[{ }^{x} \mathbf{R}^{x} \mathbf{R}^{z} \mathbf{P}_{a}\right]^{y} R, \\
\left.\left[{ }^{x} \mathbf{R}^{z} \mathbf{P}_{a}\right]^{x y} \mathbf{U},\left[{ }^{z} \mathbf{P}^{y} \mathbf{P}_{a}\right]{ }^{x y} \mathbf{U},\left[{ }^{x} \mathbf{R}^{y} \mathbf{P}_{a}^{z} \mathbf{P}\right]^{y} R,\left[{ }^{x} \mathbf{R}^{y} \mathbf{P}_{a}^{z} \mathbf{P}_{a}\right]\right]^{y} R,\left[{ }^{z} \mathbf{P}_{a}^{y} \mathbf{P}_{a}\right]{ }^{x y} \mathbf{U}\end{array}$ \\
\hline$\chi(y)$ & $\begin{array}{l}{\left[{ }^{y} \mathbf{R}^{y} \mathbf{R}^{y} \mathbf{R}^{y} P\right],\left[{ }^{y} \mathbf{R}^{y} \mathbf{R}^{y} \mathbf{R}^{y} P_{a}\right],\left[{ }^{y} \mathbf{R}^{y} \mathbf{R}^{y} \mathbf{C}\right],\left[{ }^{x} \mathbf{P}^{y} \mathbf{R}^{y} \mathbf{R}^{y} P\right],\left[{ }^{x} \mathbf{P}^{y} \mathbf{R}^{y} \mathbf{R}^{y} P_{a}\right],\left[{ }^{x} \mathbf{P}_{a}{ }^{y} \mathbf{R}^{y} \mathbf{R}^{y} P\right],} \\
{\left[{ }^{x} \mathbf{P}_{a}{ }^{y} \mathbf{R}^{y} \mathbf{R}^{y} P_{a}\right],\left[{ }^{x} \mathbf{P}^{y} \mathbf{R}^{y} \mathbf{C}\right],\left[{ }^{x} \mathbf{P}_{a}^{y} \mathbf{R}^{y} \mathbf{C}\right],\left[{ }^{x} \mathbf{P}^{z} \mathbf{P}^{y} \mathbf{R}^{y} P\right],\left[{ }^{x} \mathbf{P}^{z} \mathbf{P}_{a}^{y} \mathbf{R}^{y} P\right],\left[{ }^{x} \mathbf{P}^{z} \mathbf{P}^{y} \mathbf{R}^{y} P_{a}\right],} \\
{\left[{ }^{x} \mathbf{P}^{z} \mathbf{P}_{a}^{y} \mathbf{R}^{y} P_{a}\right],\left[{ }^{x} \mathbf{P}_{a}^{z} \mathbf{P}_{a}^{y} \mathbf{R}^{y} P_{a}\right],\left[{ }^{x} \mathbf{P}^{z} \mathbf{P}^{y} \mathbf{C}\right],\left[{ }^{x} \mathbf{P}^{z} \mathbf{P}_{a}^{y} \mathbf{C}\right],\left[{ }^{x} \mathbf{P}_{a}^{z} \mathbf{P}^{y} \mathbf{C}\right],\left[{ }^{x} \mathbf{P}_{a}^{z} \mathbf{P}_{a}^{y} \mathbf{C}\right]}\end{array}$ \\
\hline
\end{tabular}

Note. (1) $R, P, C$, and $U$ stand for revolute, prismatic, cylindrical, and universal joint and $P_{a}$ stands for parallelogram loop pair. (2) The notation of black body represents the planar joints. (3) The internal joint of [] can be arbitrary in order.

\subsection{Selecting Limbs of 2T1R DPMs Based on Screw Theory}

Limb 1. The DOF of limb is equal to 5, which contains one constraint force; that is,

$$
h_{r}=0 \quad \text { (pitch of the wrench). }
$$

The kinematic pair (KP for short) screw is a fifth-order screw system. All screws of limb are reciprocal with the constraint wrench.

(1) For revolute pairs, $h=0$. Based on reciprocal screw theory, the condition is

$$
\begin{aligned}
a & =0 \\
\text { or } \sin \alpha & =0,
\end{aligned}
$$

where $a$ is the common normal of two axes and $\alpha$ is the intersection angle of two axes.

The axes of the revolute pairs must be parallel or intersecting with the constraint wrench in the KP screw system.

(2) For prismatic pairs, $h=\infty$. Based on reciprocal screw theory, the condition is

$$
\not \cdot \phi^{r}=\cos \alpha=0 \text {. }
$$

The directions of the prismatic pairs must be perpendicular to the constraint wrench. The constraint force is

$$
\oint_{r}^{l 1}=(0,0,1 ; 0,0,0) .
$$

From (24) (26) and the criteria in Section 3.2, the orthogonal basis $\left(\mathbf{s}_{1}^{m}\right)$ of the KP screw systems is

$$
\mathbf{s}_{1}^{m}=\left\{\begin{array}{l}
s_{1}=(1,0,0 ; 0,0,0) \\
s_{2}=(0,1,0 ; 0,0,0) \\
s_{3}=(0,0,1 ; 0,0,0) \\
s_{4}=(0,0,0 ; 1,0,0) \\
s_{5}=(0,0,0 ; 0,1,0) .
\end{array}\right.
$$

TABLE 2: Equivalent limbs of one constraint force.

\begin{tabular}{lcc}
\hline Generator & $\begin{array}{c}\text { Equivalent limbs with } \\
\text { simple joints }\end{array}$ & $\begin{array}{c}\text { Equivalent limbs with } \\
\text { complex joints }\end{array}$ \\
\hline$T_{2}(x) \cdot \mu(N, x, y)$ & - & - \\
$D_{2}^{3}(N, x, y)$ & ${ }^{z} \mathbf{R}^{z} \mathbf{R}^{z} \mathbf{R}^{y} R^{y} R$ & ${ }^{z} \mathbf{R}^{z y} \mathbf{U}^{z y} \mathbf{U}$ \\
& ${ }^{z} \mathbf{R}^{z} \mathbf{R}^{z} \mathbf{R}\left[P^{y} R\right]$ & ${ }^{z} \mathbf{R}^{z} \mathbf{R}^{z y} \mathbf{U}^{z} P$ \\
\hline
\end{tabular}

The KP screw systems that satisfy (27) in Table 1 are listed in Table 2.

Limb 2. The DOF of limb is equal to 4 , which contains one constraint force and one constraint couple; that is,

$$
\begin{aligned}
& h_{r 1}=o ; \\
& h_{r 2}=\infty .
\end{aligned}
$$

The KP screw is a fourth-order screw system. All screws of limb are reciprocal with the constraint wrench.

(1) For revolute pairs, $h=0$. Based on reciprocal screw theory, the condition is

$$
\begin{aligned}
\cos \alpha_{1} & =0, \\
a \cos \alpha_{2} & =0,
\end{aligned}
$$

where $\alpha_{i}(i=1,2)$ is the intersection angle of two axes.

(2) For prismatic pairs, $h=\infty$. Based on reciprocal screw theory, the axes of the prismatic pairs must be linearly independent.

The limbs contain one constraint force and one constraint couple is expressed as follows:

$$
\oiint_{r}^{l 2}=\left\{\begin{array}{l}
\oiint_{21}^{l r}=(0,0,1 ; 0,0,0) \\
\oiint_{22}^{l r}=(0,0,0 ; 0,1,0)
\end{array}\right.
$$


TABLE 3: Equivalent limbs of one constraint force and one couple.

\begin{tabular}{lcc}
\hline Generator & $\begin{array}{c}\text { Equivalent limbs with } \\
\text { simple joints }\end{array}$ & $\begin{array}{c}\text { Equivalent limbs with } \\
\text { complex joints }\end{array}$ \\
\hline$g(x) \cdot R(N, x)$ & - & - \\
$\chi(y)$ & ${ }^{y} \mathbf{R}^{y} \mathbf{R}^{y} \mathbf{R}^{y} P$ & ${ }^{y} \mathbf{R}^{y} \mathbf{R}^{y} C$ \\
& ${ }^{x} \mathbf{P}^{y} \mathbf{R}^{y} \mathbf{R}^{y} P$ & ${ }^{x} \mathbf{P}^{y} \mathbf{R}^{y} C$ \\
\hline
\end{tabular}

From (29) (30) and the criteria in Section 3.2, the orthogonal basis $\left(\mathbf{s}_{2}^{m}\right)$ of the KP screw system can be obtained as follows:

$$
\mathbf{s}_{2}^{m}=\left\{\begin{array}{l}
s_{1}=(1,0,0 ; 0,0,0) \\
s_{2}=(0,0,1 ; 0,0,0) \\
s_{3}=(0,0,0 ; 1,0,0) \\
s_{4}=(0,0,0 ; 0,1,0)
\end{array}\right.
$$

The KP screw systems that satisfy (31) in Table 1 are listed in Table 3.

Limb 3. The DOF of limb is 3, which contains one constraint force and two constraint couples. That is,

$$
\begin{aligned}
& h_{r 1}=0 ; \\
& h_{r 2}=h_{r 3}=\infty .
\end{aligned}
$$

The KP screw is a third-order screw system. All screws of limb are reciprocal with the constraint wrench.

(1) For revolute pairs, $h=0$. Based on reciprocal screw theory, the condition is

$$
\begin{aligned}
a \sin \alpha_{1} & =0, \\
\cos \alpha_{2} & =0, \\
\cos \alpha_{3} & =0,
\end{aligned}
$$

where $\alpha_{i}(i=1,2,3)$ is the intersection angle of two axes.

(2) For prismatic pairs, $h=h_{r}=\infty$. Based on reciprocal screw theory, the condition is

$$
\begin{aligned}
\cos \alpha_{1} & =0, \\
\cos \alpha_{2} & =0, \\
a \sin \alpha_{3} & =0 .
\end{aligned}
$$

The limbs contain one constraint force and two constraint couples are expressed as follows:

$$
\oiint_{r}^{l 3}=\left\{\begin{array}{l}
\oiint_{31}^{l r}=(0,0,1 ; 0,0,0) \\
\oiint_{32}^{l r}=(0,0,0 ; 0,1,0) \\
\oiint_{33}^{l r}=(0,0,0 ; 0,0,1)
\end{array}\right.
$$

TABLE 4: Equivalent limbs with one constraint force and two couples.

\begin{tabular}{lcc}
\hline Generator & $\begin{array}{c}\text { Equivalent limbs with } \\
\text { simple joints }\end{array}$ & $\begin{array}{c}\text { Equivalent limbs with } \\
\text { complex joints }\end{array}$ \\
\hline$T_{2}(x) \cdot R(N, y)$ & ${ }^{x} P^{y} R^{y} P$ & - \\
\hline
\end{tabular}

TABLE 5: The configuration of 2TIR DPMs.

\begin{tabular}{lcc}
\hline$N$ & Combination form & Number of configurations \\
\hline 1 & $L_{1} \cup L_{2} \cup L_{3}$ & 4 \\
2 & $L_{1} \cup L_{1} \cup L_{3}$ & - \\
3 & $L_{2} \cup L_{2} \cup L_{3}$ & 3 \\
\hline
\end{tabular}

Note. $L_{i}$ is the $i$ th limb.

From (33) (35) and the criteria in Section 3.2, the orthogonal basis $\left(\mathbf{s}_{3}^{m}\right)$ of the KP screw system is

$$
\mathbf{s}_{3}^{m}=\left\{\begin{array} { l } 
{ a \operatorname { s i n } \alpha _ { 1 } = 0 } \\
{ \operatorname { c o s } \alpha _ { 2 } = 0 } \\
{ \operatorname { c o s } \alpha _ { 3 } = 0 }
\end{array} \cap \left\{\begin{array}{l}
\cos \alpha_{1}=0 \\
\cos \alpha_{2}=0 \\
a \sin \alpha_{3}=0
\end{array}\right.\right.
$$

The KP screw systems that satisfy (36) in Table 1 are listed in Table 4.

4.5. Type Synthesis of 2T1R DPMs. According to screw theory, the reciprocal screw system of $2 \mathrm{~T} 1 \mathrm{R}$ DPMs is

$$
\boldsymbol{S}_{M}=\oiint_{1}^{m} \cap \oiint_{2}^{m} \cap \oiint_{3}^{m}=\mathbf{s}_{1}^{m} \cap \mathbf{s}_{2}^{m} \cap \mathbf{s}_{3}^{m} .
$$

The configuration of 2T1R DPMs with basic complex joints (the mechanism only with simple joints are no actual use) can be constituted as Table 5 .

The rotational axis of the revolute DOF is parallel to the plane composed of the translational axes, called spatial 2T1R DPMs.

According to Table 5, spatial 2T1R DPMs are presented in Figures 3 and 4 , with the notation of $R_{i j}$, where $i$ represents the number of limbs and $j$ represents the order of the joint. The underlined is the driving pair, and the joint in bracket is driving pair of the complex pair.

In Figure 3, the moving platform (MP) is connected by three limbs whose DOFs are different. The relationship of the subspace of $L_{i}$ is

$$
\mathbf{s}_{1}^{m} \supset \mathbf{s}_{2}^{m} \supset \mathbf{s}_{3}^{m} \text {. }
$$

The MP has three independent motions (2 translations and 1 rotation). In Figure 3(a), the rotation of the MP depends on driving pair $R_{11}$, and the translations of the MP depend on driving pairs $P_{21}$ and $P_{31}$. In Figure 3(b), the rotation of the MP depends on driving pair $R_{11}$, the translations of the MP depend on driving pairs $P_{21}$ and $P_{32}$. In Figure 3(c), the rotation of the MP depends on driving pair $P_{11}$, and the translations of the MP depend on driving pairs $P_{21}$ and $P_{31}$. In Figure 3(d), the rotation of the MP depends on driving pair $P_{11}$, and the translations of the MP depend on driving pairs $P_{21}$ and $P_{32}$. The configuration is completely decoupled. 


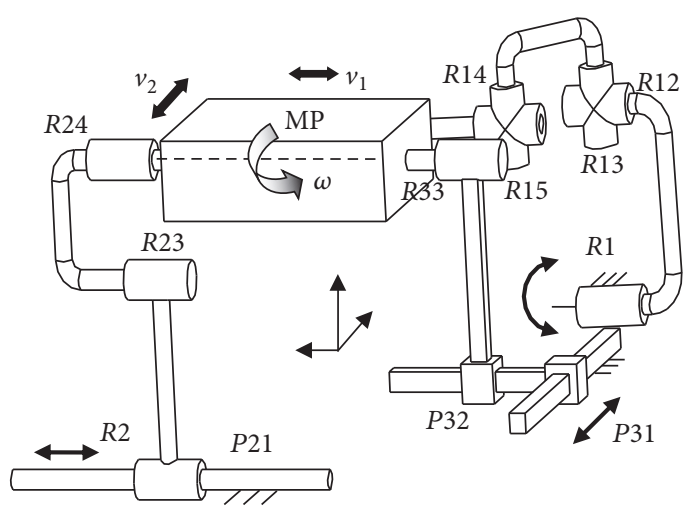

(a) $U U-C(\underline{P}) R R-\underline{P} P R$

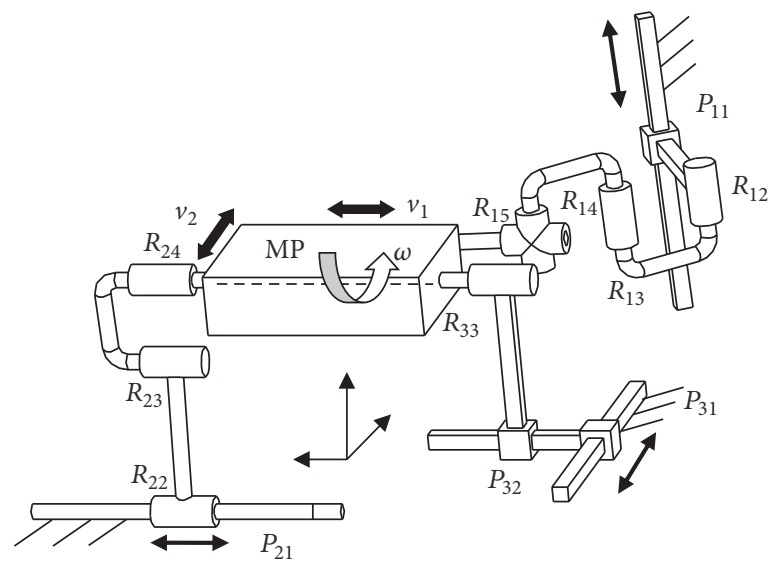

(c) $\underline{P} R R U-C(\underline{P}) R R-\underline{P} P R$

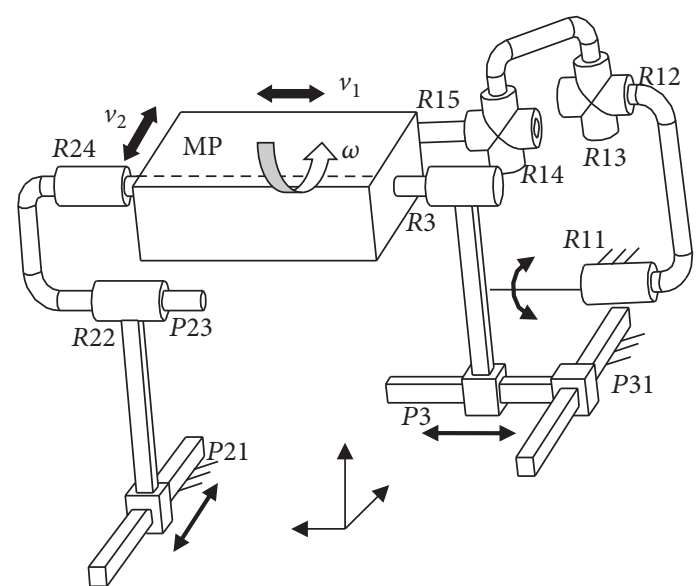

(b) $\underline{R} U U-\underline{P} C R-\underline{P} P R$

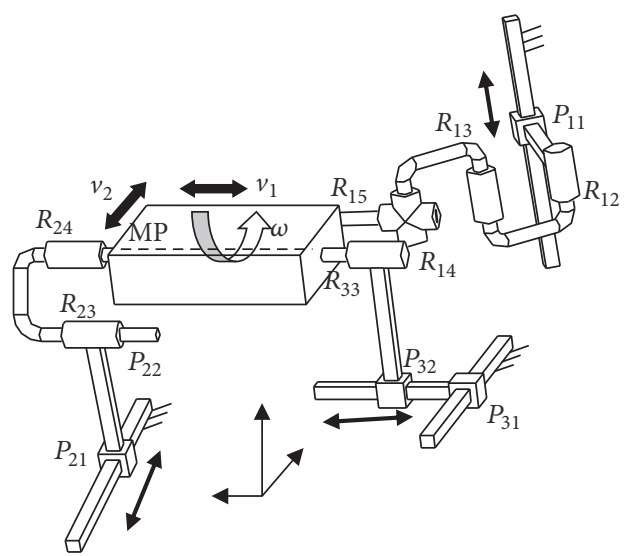

(d) $\underline{P} R R U-\underline{P} C R-\underline{P} P R$

FIGURE 3: Spatial 2T1R DPMs.

Figures 3(a) and 3(c) show two optimal configurations, in which the moving inertia of the mechanism is smallest for the actuators close to the base.

In Figure 4, the moving platform (MP) is connected by three limbs in which $L_{1}$ and $L_{2}$ have the same DOF. The relationship of the subspace of $L_{i}$ is

$$
\mathbf{s}_{1}^{m}=\mathbf{s}_{2}^{m} \supset \mathbf{s}_{3}^{m} \text {. }
$$

The MP has three independent motions (2 translations and 1 rotation). In Figure 4(a), the rotation of the MP depends on driving pair $R_{21}$, the translations of the MP depend on driving pairs $P_{11}$ and $P_{32}$. In Figure 4(b), the rotation of the MP depends on driving pair $R_{11}$, and the translations of the MP depend on driving pairs $P_{21}$ and $P_{32}$. In Figure 4(c), the rotation of the MP depends on driving pair $R_{11}$, and the translations of the MP depend on driving pairs $P_{21}$ and $P_{31}$. The configuration is decoupled.

Figure 4(c) shows a design with actuators located on the base, which reduces the moving inertia of the mechanism.

The rotational axis of the revolute DOF is vertical to the plane composed of the translational axes, called planar 2T1R DPMs.
According to the method introduced here, the planar 2T1R DPMs will be derived. The results are shown in Figures 5 and 6.

In Figure 5, the moving platform (MP) is connected by three limbs whose DOFs are different. The relationship of the subspace of $L_{i}$ is

$$
\mathbf{s}_{1}^{m} \supset \mathbf{s}_{2}^{m} \supset \mathbf{s}_{3}^{m} .
$$

The MP has three independent motions (2 translations and 1 rotation). The rotation of the MP depends on driving pair $R_{11}$, and the translations of the MP depend on driving pairs $P_{21}$ and $P_{31}$. The configuration is decoupled. The driving pair is installed in the fixed platform. The moving inertia of the mechanism is smallest.

In Figure 6, the moving platform (MP) is connected by three limbs in which $L_{1}$ and $L_{2}$ have the same DOF. The relationship of the subspace of $L_{i}$ is

$$
\mathbf{s}_{1}^{m}=\mathbf{s}_{2}^{m} \supset \mathbf{s}_{3}^{m} .
$$

The MP has three independent motions (2 translations and 1 rotation). In Figure 6(a), the rotation of the MP depends on driving pair $R_{11}$, and the translations of the MP depend on 


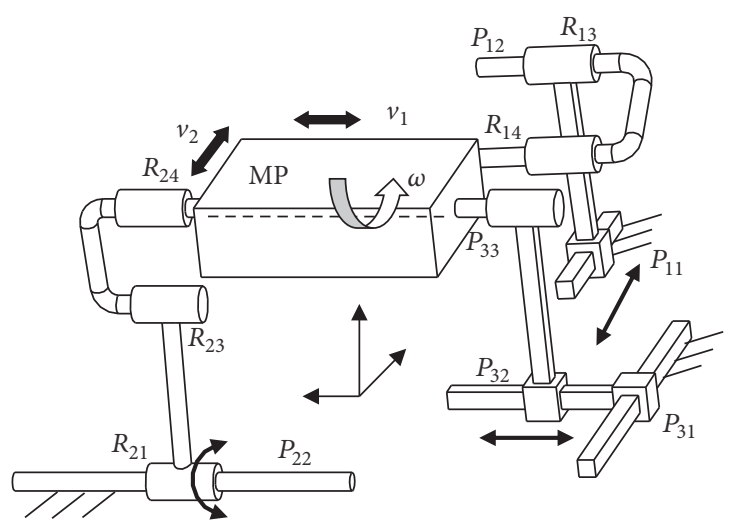

(a) $\underline{P} C R-C(\underline{R}) P R R-\underline{P} P R$

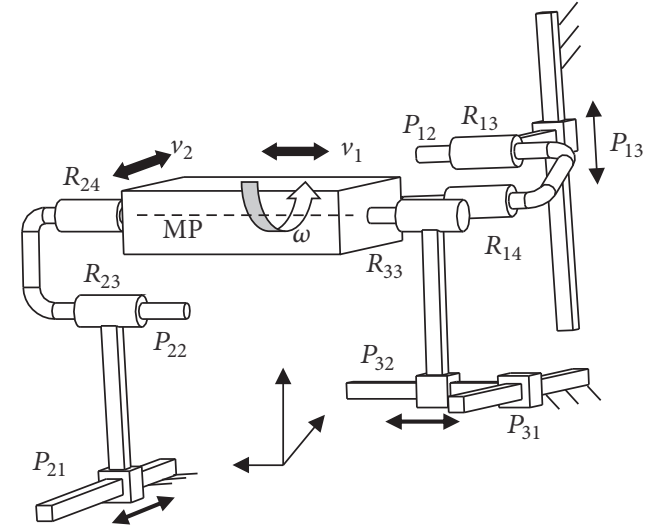

(b) $\underline{P P R R}-\underline{P} C R-\underline{P} P R$

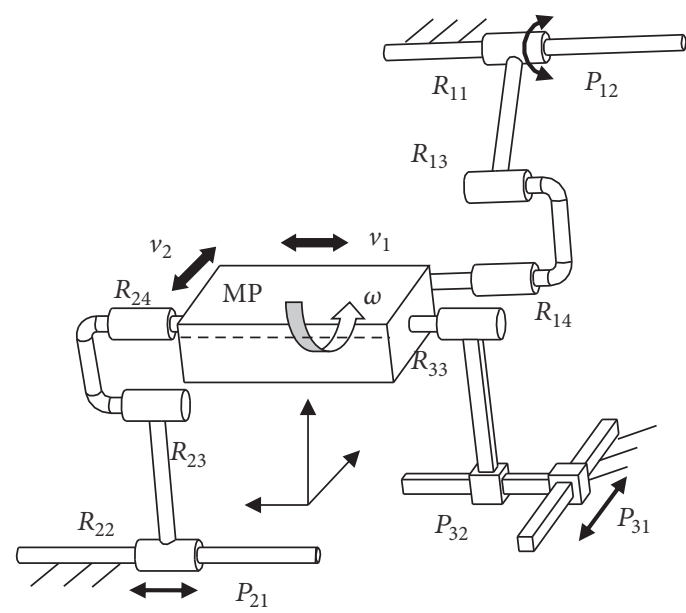

(c) $C(\underline{R}) R R-C(\underline{P}) R R-\underline{P} P R$

Figure 4: Spatial 2T1R DPMs.

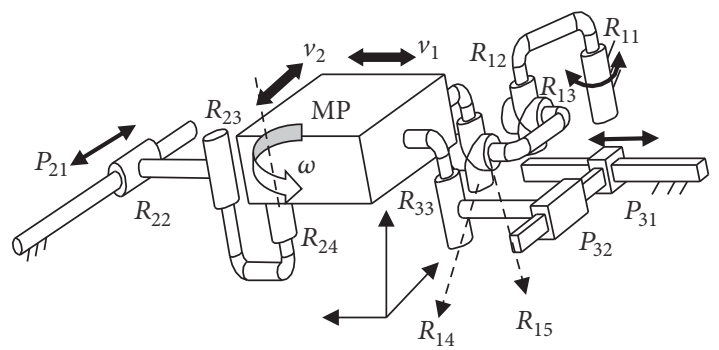

(a) $\underline{P} U U-C(\underline{P}) R R-\underline{P} P R$

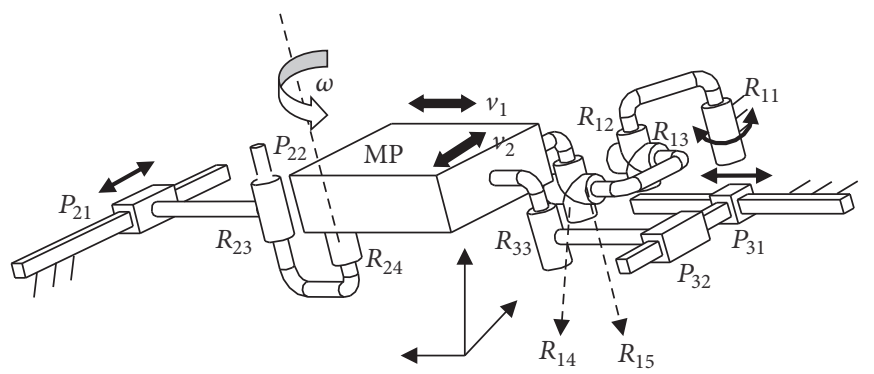

(b) $\underline{R} U U-\underline{P} C R-\underline{P} P R$

Figure 5: Planar 2T1R DPMs.

driving pairs $P_{21}$ and $P_{31}$. In Figures 6(b) and 6(c) the rotation of the MP depends on driving pair $R_{21}$, and the translations of the MP depend on driving pairs $P_{11}$ and $P_{31}$.

Similarly, the driving pair is installed in the fixed platform. The moving inertia of the mechanism is smallest.

In total, 12 parallel mechanisms with decoupled 2T1R motions were synthesized. To the authors' best knowledge, this is the first time that these mechanisms are synthesized and reported.

\section{Analysis of the Synthesized 2T1R DPM}

Figure 7 shows one of the spatial 2T1R DPMs (Figure 3(c)). According to the modified Kutzbach-Grübler criterion [28], the DOF of the spatial parallel mechanism is readily found as 3.

As shown in Figure 7, there are two prismatic pairs $\left(P_{1}, P_{3}\right)$ and cylindrical pair $C_{2}$ is the driving pair. $H$ is one point of line $l$ on the moving platform. $q_{i}(i=1,2,3)$ is the 


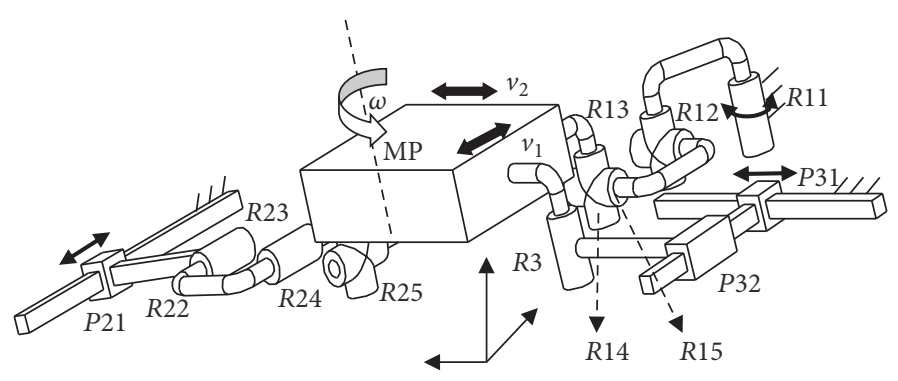

(a) $\underline{R} U U-\underline{P} R R U-\underline{P} P R$

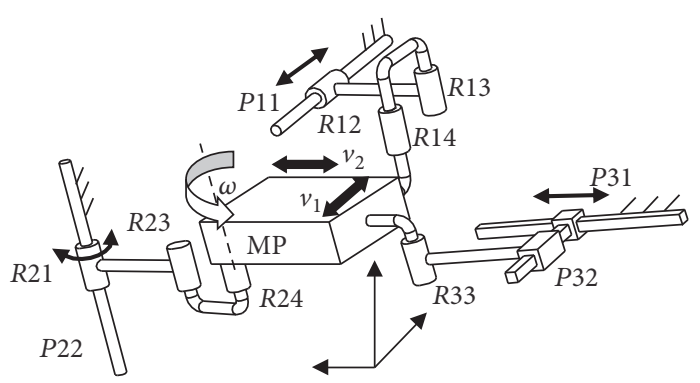

(b) $C(P) R R-C(R) R R-P P R$

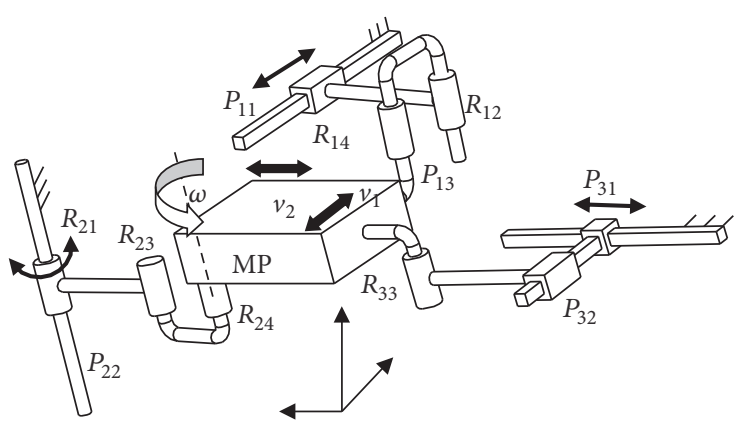

(c) $P C R-C(R) R R-P P R$

FIgURE 6: Planar 2T1R DPMs.

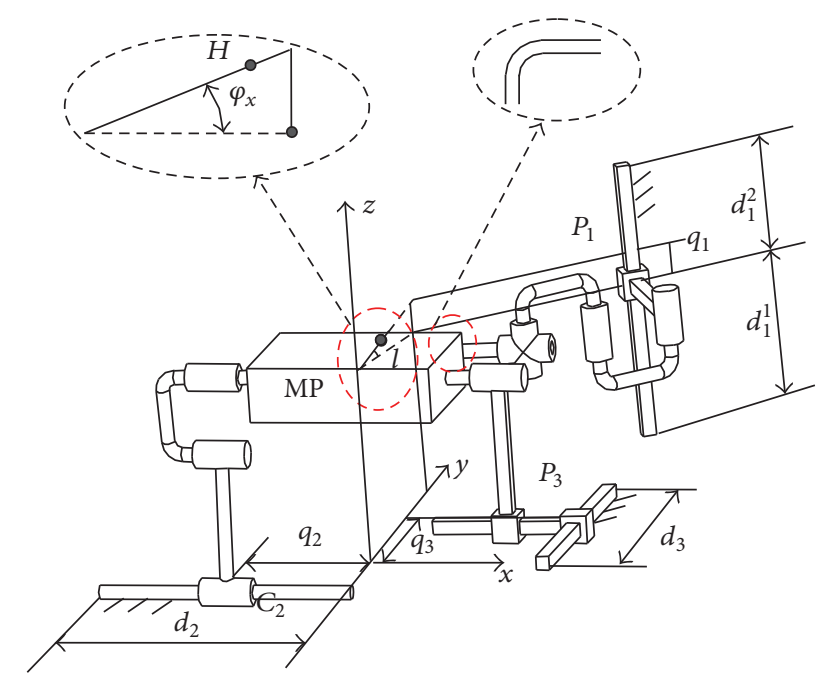

FIgURE 7: One of spatial 2T1R DPMs.

moving distance of the driving pairs. $d_{i}(i=1,2,3)$ is the moving range of $P_{1}, C_{2}$, and $P_{3}$, where $d_{1}=d_{1}^{1}+d_{1}^{2}$.

The kinematic equation is

$$
\left[\begin{array}{c}
H_{\varphi_{x}} \\
H_{x} \\
H_{y}
\end{array}\right]=\left[\begin{array}{c}
\arctan \frac{p_{1}}{l} \\
p_{2} \\
p_{3}
\end{array}\right] .
$$

Differentiating (42) with respect to time yields

$$
\left[\begin{array}{c}
\dot{H}_{\varphi_{x}} \\
\dot{H}_{x} \\
\dot{H}_{y}
\end{array}\right]=\left[\begin{array}{ccc}
\frac{l}{1+p_{1}^{2}} & 0 & 0 \\
0 & 1 & 0 \\
0 & 0 & 1
\end{array}\right]\left[\begin{array}{c}
\dot{p}_{1} \\
\dot{p}_{2} \\
\dot{p}_{3}
\end{array}\right] .
$$

$J_{a}$ is a diagonal matrix with different diagonal element. The PM is a decoupled mechanism in the whole workspace.

\section{Conclusions}

This paper presents a new design method to synthesize DPMs by virtue of Lie groups and screw theory. The method is developed by synthesizing separately at limb and configuration levels. At limb level, Lie group is used to synthesize the limbs with required DOFs. At configuration level, the screw theory is adopted to determine configurations with synthesized limbs that satisfy the type synthesis criteria of DPMs. A number of constraints on the joint axes are analyzed and formulated.

With the synthesis method, type synthesis was conducted, which leads to totally 12 decoupled parallel mechanisms for $2 \mathrm{~T} 1 \mathrm{R}$ motions. Of these mechanisms, seven are able to produce spatial motions, while the remaining five produce planar decoupled motions. This is the first time these mechanisms are synthesized, which is a major contribution of this work. All the mechanisms can be considered for their practical applications of engineering.

The proposed synthesis method is based on the orthogonal conditions of limbs, namely, a set of constraints of joint 
axes in the kinematic chain of a limb. Generally, all decoupled mechanisms have to be synthesized with orthogonal conditions. For this work, these conditions are established for the 2T1R motion. When the method is extended for other types of motions, particularly for motions with more degrees of freedom, the conditions have to be checked and validated.

\section{Conflicts of Interest}

The authors declare that there are no conflicts of interest regarding the publication of this paper.

\section{Acknowledgments}

This research is supported by National Natural Science Foundation of China (Grant no. 51275486) and Graduate Innovation Project of Shanxi Province (Grant no. 2016BY126).

\section{References}

[1] D. Zeng, Y. Hou, W. Lu, and Z. Huang, "Comparative analysis of characteristics of the coupled and decoupled parallel mechanisms," Chinese Journal of Mechanical Engineering (English Edition), vol. 23, no. 4, pp. 468-476, 2010.

[2] G. Gogu, "Structural synthesis of fully-isotropic translational parallel robots via theory of linear transformations," European Journal of Mechanics, A/Solids, vol. 23, no. 6, pp. 1021-1039, 2004.

[3] G. Gogu, "Structural synthesis of maximally regular T3R2type parallel robots via theory of linear transformations and evolutionary morphology," Robotica, vol. 27, no. 1, pp. 79-101, 2009.

[4] G. Gogu, “T2R1-type parallel manipulators with bifurcated planar-spatial motion," European Journal of Mechanics, A/Solids, vol. 33, pp. 1-11, 2012.

[5] Q. Jin and T.-L. Yang, "Synthesis and analysis of a group of 3 degree-of-freedom partially decoupled parallel manipulators," Journal of Mechanical Design, Transactions of the ASME, vol. 126, no. 2, pp. 301-306, 2004.

[6] Z. Huang and Q. C. Li, 3-DOF translational parallel robot mechanism, China Patent, 2001.

[7] V. Glazunov, "Design of decoupled parallel manipulators by means of the theory of screws," Mechanism and Machine Theory, vol. 45, no. 2, pp. 239-250, 2010.

[8] D. Zeng and Z. Huang, "Type synthesis of the rotational decoupled parallel mechanism based on screw theory," Science China Technological Sciences, vol. 54, no. 4, pp. 998-1004, 2011.

[9] Q. Chen, Q. Li, C. Wu, and Y. Li, "Mobility, constraint singularity and isotropy of the translational parallel mechanism," Chinese Journal of Mechanical Engineering (English Edition), vol. 22, no. 6, pp. 841-848, 2009.

[10] J. Gallardo-Alvarado, A. Ramírez-Agundis, H. Rojas-Garduño, and B. Arroyo-Ramírez, "Kinematics of an asymmetrical threelegged parallel manipulator by means of the screw theory," Mechanism and Machine Theory, vol. 45, no. 7, pp. 1013-1023, 2010.

[11] D. X. Zeng, Z. T. Hu, and Y. L. Hou, "Type synthesis of 2R1T decoupled parallel mechanism based on screw theory," Journal of Yanshan University, vol. 38, no. 1, pp. 22-28, 2014.

[12] Y.-G. Huang and M.-L. Huang, "Synthesis of planar 2T1R nonoverconstrained parallel mechanisms," Journal of Chongqing University, vol. 35, no. 5, pp. 481-486, 2009.
[13] W. Li, F. Gao, and J. Zhang, "R-CUBE, a decoupled parallel manipulator only with revolute joints," Mechanism and Machine Theory, vol. 40, no. 4, pp. 467-473, 2005.

[14] Y. Jin, I.-M. Chen, and G. Yang, "Kinematic design of a family of 6-DOF partially decoupled parallel manipulators," Mechanism and Machine Theory, vol. 44, no. 5, pp. 912-922, 2009.

[15] S. Briot and I. A. Bonev, "Pantopteron-4: a new 3T1R decoupled parallel manipulator for pick-and-place applications," Mechanism and Machine Theory, vol. 45, no. 5, pp. 707-721, 2010.

[16] J. Gallardo-Alvarado, H. Orozco-Mendoza, and J. M. RicoMartínez, "A novel five-degrees-of-freedom decoupled robot," Robotica, vol. 28, no. 6, pp. 909-917, 2010.

[17] R. Vertechy and V. Parenti-Castelli, "Kinematic analysis of partially decoupled fully-parallel manipulators of type 5-5 and 4-5," Robotica, vol. 27, no. 2, pp. 235-240, 2009.

[18] O. Altuzarra, M. Loizaga, C. Pinto, and V. Petuya, "Synthesis of partially decoupled multi-level manipulators with lower mobility," Mechanism and Machine Theory, vol. 45, no. 1, pp. 106-118, 2010.

[19] F. Xie, X.-J. Liu, Z. You, and J. Wang, “Type synthesis of 2T1Rtype parallel kinematic mechanisms and the application in manufacturing," Robotics and Computer-Integrated Manufacturing, vol. 30, no. 1, pp. 1-10, 2014.

[20] Y. B. Zhang, S. Q. Zhang, and X. Wu. Design, "kinematics analysis of a 2T1R-type spatial parallel robotic mechanisms," Mechanical Science and Technology for Aerospace Engineering, vol. 33, no. 4, pp. 484-489, 2014.

[21] J. M. Hervé, "Analyse structurelle des mécanismes par groupe des déplacements," Mechanism and Machine Theory, vol. 13, no. 4, pp. 437-450, 1978.

[22] C. Fan, H. Liu, and Y. Zhang, "Type synthesis of 2T2R, 1T2R and 2R parallel mechanisms," Mechanism and Machine Theory, vol. 61, pp. 184-190, 2013.

[23] F. Gao, J. L. Yang, and Q. D. Ge, Type Synthesis of Parallel Mechanisms of $G_{f}$ Sets Theory, Science Press, Beijing, China, 2011.

[24] T. L. Yang, A. X. Liu, and Y. F. Luo, Theory and Application of Robot Mechanism Topology, Science Press, Beijing, China, 2012.

[25] J. S. Dai, Screw Algebra and Lie Groups and Lie Algebras, Springer, London, UK, 2014.

[26] J. J. Yu, X. J Liu, X. L. Ding et al., Mathematical Foundation of Robot Mechanisms, China Machine Press, Beijing, China, 2th edition, 2014.

[27] J. Yang, F. Gao, Q. J. Ge, X. Zhao, W. Guo, and Z. Jin, “Type synthesis of parallel mechanisms having the first class GF sets and one-dimensional rotation," Robotica, vol. 29, no. 6, pp. 895902, 2011.

[28] Y. Li, L. Wang, J. Liu, and Z. Huang, "Applicability and generality of the modified Grübler-Kutzbach criterion," Chinese Journal of Mechanical Engineering (English Edition), vol. 26, no. 2, pp. 257263, 2013. 


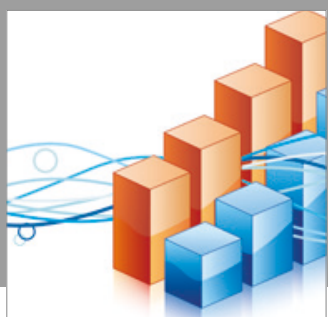

Advances in

Operations Research

vatersals

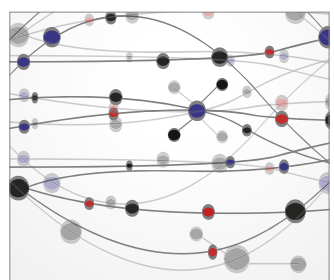

\section{The Scientific} World Journal
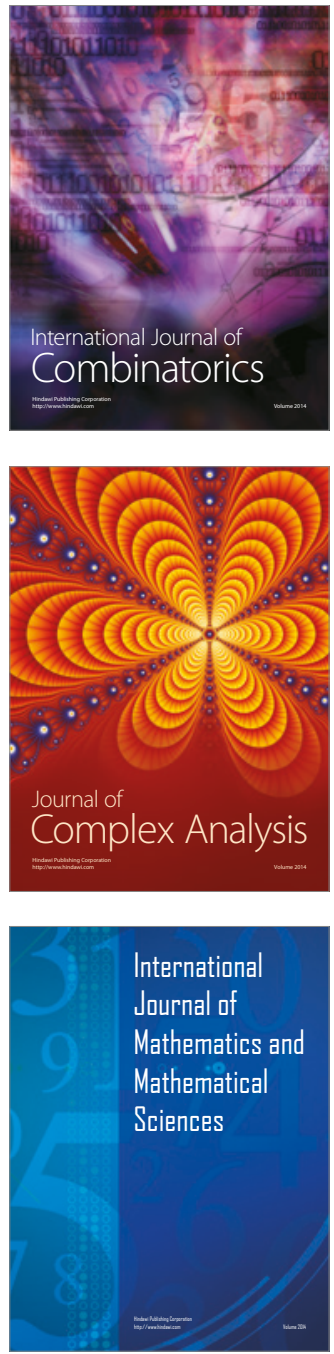
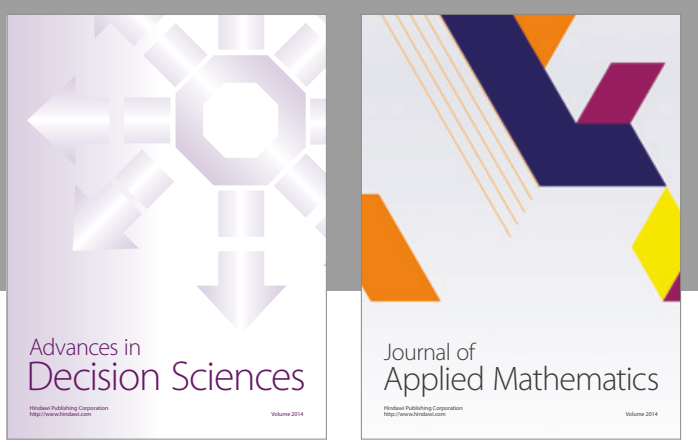

Algebra

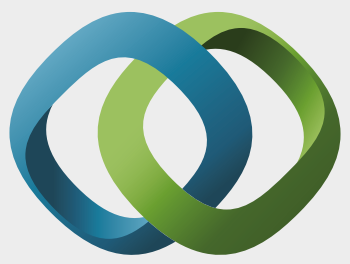

\section{Hindawi}

Submit your manuscripts at

https://www.hindawi.com
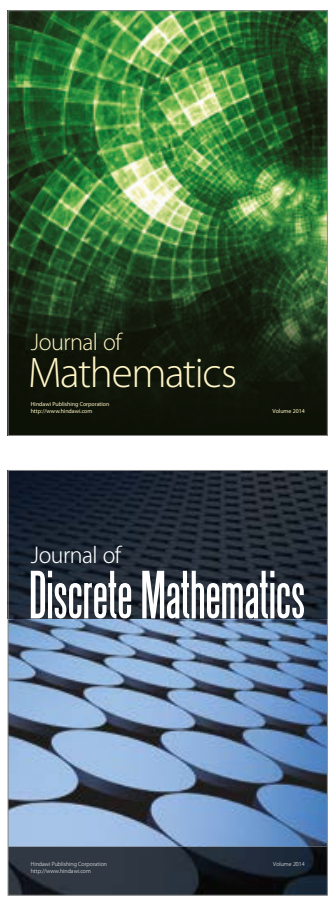

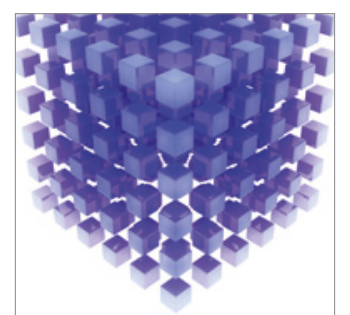

Mathematical Problems in Engineering
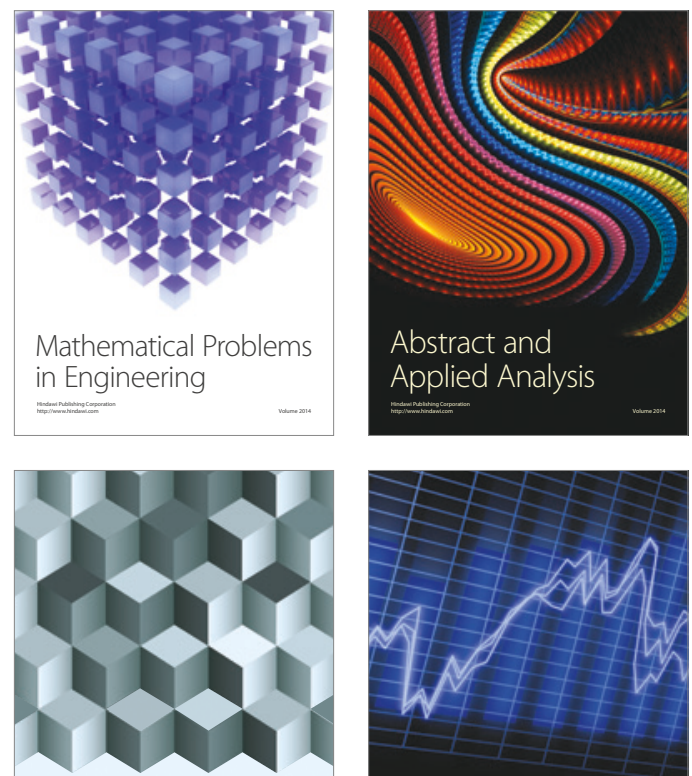

Journal of

Function Spaces

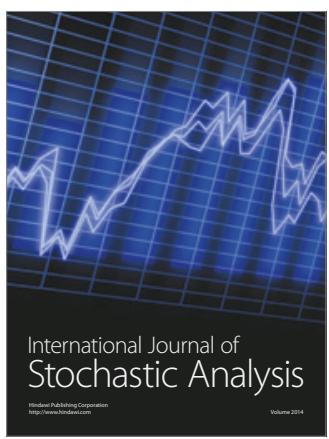

Probability and Statistics
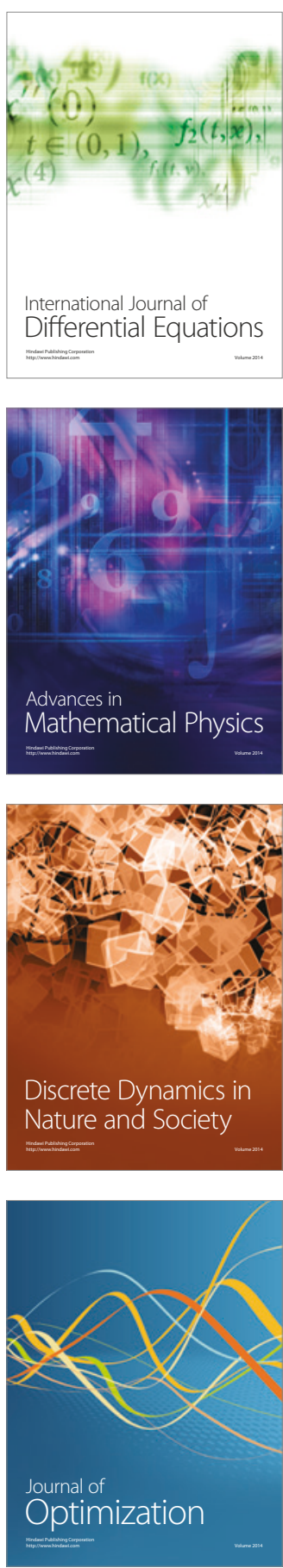